\title{
Reducing agents induce microtubule extrusion in demembranated mammalian spermatozoa
}

\author{
Masashi Kinukawa, Masao Nagata and Fugaku Aoki \\ Department of Integrated Biosciences, Graduate School of Frontier Sciences, University of Tokyo, Kashiwa, \\ Chiba 277-8562, Japan
}

Correspondence should be addressed to F Aoki; Email: aokif@k.u-tokyo.ac.jp

\begin{abstract}
To understand the mechanism regulating flagellar bending in spermatozoa, it is important to investigate the regulation of microtubule sliding in the flagellar axoneme. It has been shown that protease treatment following demembranation with Triton X-100 disrupts the connections between microtubules and induces extrusion of microtubules from the flagellar axoneme. This approach enables a direct investigation of the regulation of microtubule sliding; however, the percentage of spermatozoa with protease-induced extrusion was relatively low, probably due to protease digestion of some regulatory motility proteins, as well as proteins connecting the microtubules. In this study, we demonstrate microtubule extrusion in most hamster and mouse demembranated spermatozoa upon treatment with a high concentration of the reducing agents dithiothreitol or 2-mercaptoethanol, without the use of proteases. The extrusion of microtubules occurred when the spermatozoa were treated with concentrations of the reducing agents that were sufficient for the reduction of the disulfide bonds of IgG. These results suggest that the arrangement of the axonemal structures connecting doublet microtubules depends to an important degree on -S-S- bonds. Close observation of the extrusion process using the present method revealed that microtubules were extruded on the same side as that of the curve of the sperm head, and also on the opposite side. Furthermore, we noted that extrusion always started on one side, followed by the other side, but was never initiated on both sides simultaneously.

Reproduction (2004) 128 813-818
\end{abstract}

\section{Introduction}

The motility of spermatozoa for progressive swimming is generated by flagellar bending, which is regulated via a complex mechanism. For instance, the flagellar bending pattern of mammalian spermatozoa is changed dynamically in the genital tract after ejaculation in vivo, and in culture medium during incubation in vitro. After several hours of incubation, spermatozoa are hyperactive and the flagellar bending pattern is changed markedly from the pattern at the start of incubation; the amplitude and asymmetry of flagellar bending increases with duration of incubation (Katz et al. 1986, Suarez 1988, Suarez et al. 1991, Aoki et al. 1994). The changes occur in various ways in different parts of the flagellum, which suggests that flagellar bending is differentially regulated in different regions of the flagellum (Kinukawa et al. 2003). Thus, the mechanism that regulates flagellar bending is complex and remains to be further elucidated.

To shed light on the mechanism of activation of the flagellar axoneme, it is necessary to examine individual microtubule sliding. The typical structure of the axoneme in most eukaryotic flagella is that of a central pair of singlet microtubules, which are surrounded by nine doublet microtubules $(9+2$ arrangement). Flagellar bending is generated by the sliding of adjacent doublet microtubules via the activity of dynein arm ATPases, using $\mathrm{Mg}^{2+}$-ATP as the substrate (Shingyoji et al. 1977). In spermatozoa that are demembranated with the nonionic detergent Triton X-100, flagellar movement that is comparable to that of intact spermatozoa can be reproduced by treatment with $\mathrm{Mg}^{2+}$-ATP (Lindemann \& Gibbons 1975). The demembranated spermatozoa model is useful for the investigation of the components required for activation of the flagellar axoneme. Microtubule sliding produced by dynein ATPase activity has been demonstrated in the demembranated spermatozoa model after partial digestion of the axoneme proteins with proteases. Treatment with an appropriate concentration of trypsin (Si \& Okuno 1993, 1995) or elastase (Ishijima et al. 2002, Nakano et al. 2003) in the presence of $\mathrm{Mg}^{2+}$-ATP induces microtubule extrusion. Electron microscopic analysis has revealed that protease treatment digests the axonemal structural proteins, thereby 
disrupting both the nexin links and the radial spokes. However, it seems likely that proteases also degrade other important regulatory components that are involved in flagellar bending. Thus, microtubule extrusion induced by protease digestion does not appear to be the best model for investigating the regulatory mechanisms of microtubule sliding.

In this study, we report a method by which microtubules are extruded efficiently from demembranated mouse and hamster spermatozoa. Microtubules were efficiently extruded by treatment with reducing agents in the absence of proteases. Using this method, the characteristics of microtubule extrusion were observed closely.

\section{Materials and Methods}

\section{Sperm preparation}

Male Syrian hamsters and male ICR mice were raised and maintained in a light-controlled room $(12 \mathrm{~h}$ light: $12 \mathrm{~h}$ dark cycle) at a constant temperature $\left(22 \pm 1^{\circ} \mathrm{C}\right)$. Sexually mature male hamsters and mice were killed by chloroform inhalation and cervical dislocation respectively, and the cauda epididymis was removed promptly. After removal of blood from the epididymal surface with physiological saline solution, the distal tubules were punctured with an 18-gauge needle in five to ten places, and the sperm mass was squeezed out into a plastic Petri dish $(35 \mathrm{~mm} \times 10 \mathrm{~mm})$ using forceps. The hamster semen was covered with mineral oil that had been pre-warmed to $37^{\circ} \mathrm{C}$, in order to prevent evaporation before use. Mouse semen was introduced directly into the demembranation medium. All of the procedures described herein were reviewed and approved by the University of Tokyo Institutional Animal Care and Use Committee, and were performed in accordance with the Guiding Principles for the Care and Use of Laboratory Animals.

\section{Procedures for the induction of microtubule extrusion}

Spermatozoa reactivation was performed using a modification of the method developed by Ishijima and Witman (1991). Sucrose and potassium glutamate were omitted from the demembranation and reactivation media, since these reagents prevent the reactivation of mouse spermatozoa. A $1 \mu \mathrm{l}$ aliquot of semen was suspended in $100 \mu \mathrm{l}$ of demembranation medium that contained $1 \mathrm{mM}$ EDTA; $50 \mathrm{mM}$ N-2-hydroxyethyl-piperazine-N-2-ethane sulphonic acid (HEPES, pH 7.9); 0.2\% (w/v) Triton X-100 Sigma; and a protease inhibitor cocktail that contained $1 \mu \mathrm{g} / \mathrm{ml}$ aprotinin, $2 \mu \mathrm{g} / \mathrm{ml}$ leupeptin, $1 \mu \mathrm{g} / \mathrm{ml}$ pepstatin, and $100 \mu \mathrm{g} / \mathrm{ml}$ phenylmethyl sulfonyl fluoride. The suspension was incubated for $30 \mathrm{~s}$ with gentle stirring at $37^{\circ} \mathrm{C}$ to dissolve the plasma membrane and mitochondrial sheath. Then, $10 \mu \mathrm{l}$ of the suspension of extracted sperm were transferred to $100 \mu \mathrm{l}$ of the reactivation medium that contained $1 \mathrm{mM}$ EDTA, $1 \mathrm{mM}$ ATP, $5 \mathrm{mM} \mathrm{MgCl}, 50 \mathrm{mM}$
HEPES ( $\mathrm{pH} 7.9$ ), and the protease inhibitor cocktail. Various concentrations of dithiothreitol (DTT), 2-mercaptoethanol (2-ME), and $2 \mu \mathrm{g} / \mathrm{ml}$ trypsin plus $1 \mathrm{mM}$ DTT were added to the reactivation medium, to induce microtubule extrusion. The protease inhibitor cocktail was excluded from the demembranation and reactivation media that were used for the trypsin treatment.

\section{Microscopic observation of spermatozoa}

After the reactivated spermatozoa were incubated at $37^{\circ} \mathrm{C}$ for more than $10 \mathrm{~min}, 10 \mu \mathrm{l}$ of each sperm suspension were placed on a glass slide pre-warmed to $37^{\circ} \mathrm{C}$ and covered with an $18 \times 24 \mathrm{~mm}$ coverslip. As soon as the sample was prepared, photomicrographs were taken at 60 frames/s and an exposure time of $1 / 1000 \mathrm{~s}$, with a FASTCAM-Net high-speed camera (Photron, Tokyo, Japan) on a phase-contrast microscope. The images obtained were recorded using Movie Ruler (Photron).

\section{Determination of the reducing power of DTT and 2-ME}

To investigate the reducing power of DTT and 2-ME, various concentrations of each agent were used to reduce $\operatorname{lgG}$, and the resulting protein conformations were analyzed by non-reducing SDS-PAGE. IgG (whole goat IgG; Jackson ImmunoResearch Laboratories Inc, West Grove, PA, USA) was added in $100 \mu \mathrm{g}$ aliquots to microcentrifuge tubes that contained $500 \mu \mathrm{l}$ of reactivation medium plus various concentrations of DTT or 2-ME. After incubation for $10 \mathrm{~min}$ at $37^{\circ} \mathrm{C}$, the proteins were precipitated with an equal volume of $10 \%(\mathrm{w} / \mathrm{v})$ trichloroacetic acid. Each sample was centrifuged at $10000 \mathrm{~g}$ for $10 \mathrm{~min}$ at $4^{\circ} \mathrm{C}$, and the supernatant was discarded. The pellet was washed with $5 \%(\mathrm{w} / \mathrm{v})$ trichloroacetic acid and again centrifuged at $10000 \mathrm{~g}$ for $10 \mathrm{~min}$ at $4{ }^{\circ} \mathrm{C}$. After discarding the supernatant, the pellet was dissolved in $25 \mu l 0.1 \mathrm{~N} \mathrm{NaOH}$ and the $\mathrm{pH}$ was adjusted by the addition of $15 \mu \mathrm{l} 0.1 \mathrm{M} \mathrm{HCl}$. Then, $40 \mu \mathrm{l} 2 \times$ SDS sample buffer (Laemmli 1970) without 2-ME were added to each sample and the mixtures were boiled for $1 \mathrm{~min}$. The samples were subjected to electrophoresis on $7.5 \%$ SDS-PAGE gels, along with prestained SDS-PAGE molecular weight standards (Bio-Rad Laboratories, Inc., Hercules, CA, USA). The gels were fixed and stained with Coomassie brilliant blue R-250. After staining, the gels were dried for $2 \mathrm{~h}$ at $80^{\circ} \mathrm{C}$.

\section{Results}

\section{Efficient induction of microtubule extrusion by reducing agents}

In order to minimize protein digestion by endogenous proteases, the protease inhibitor cocktail was added to the demembranation and reactivation media. In the presence of protease inhibitors, microtubule extrusion occurred in most hamster spermatozoa upon the addition of concentrations greater than $10 \mathrm{mM}$ DTT or $100 \mathrm{mM} 2-\mathrm{ME}$ to the 
reactivation medium (Fig. 1a); most mouse spermatozoa showed microtubule extrusion when concentrations greater than $1 \mathrm{mM}$ DTT or $33 \mathrm{mM} 2-\mathrm{ME}$ were added (Fig. 1b). Treatment with $2 \mu \mathrm{g} / \mathrm{ml}$ trypsin plus $1 \mathrm{mM}$ DTT caused microtubule extrusion in $42 \%$ of the mouse spermatozoa, whereas extrusion occurred in $82 \%$ of spermatozoa that were treated with $1 \mathrm{mM}$ DTT alone (Table 1), which indicates that trypsin has a detrimental effect on microtubule extrusion. Microtubule extrusion was not observed in hamster spermatozoa that were treated with $1 \mathrm{mM}$ DTT or trypsin plus $1 \mathrm{mM}$ DTT, whereas microtubules were extruded from most of the spermatozoa that were treated with $33 \mathrm{mM}$ DTT (Table 1).

In both the hamster and mouse spermatozoa, the concentrations of DTT and 2-ME required to induce the extrusion of microtubules differed by 10- to 33-fold (Fig. 1a, b). This difference appears to be due to the different reducing potencies of the two agents. To confirm this, we examined

(a) hamster

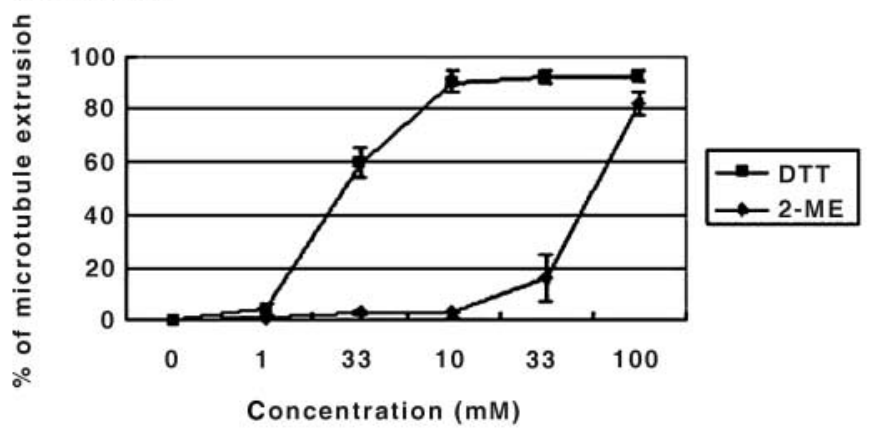

(b) mouse
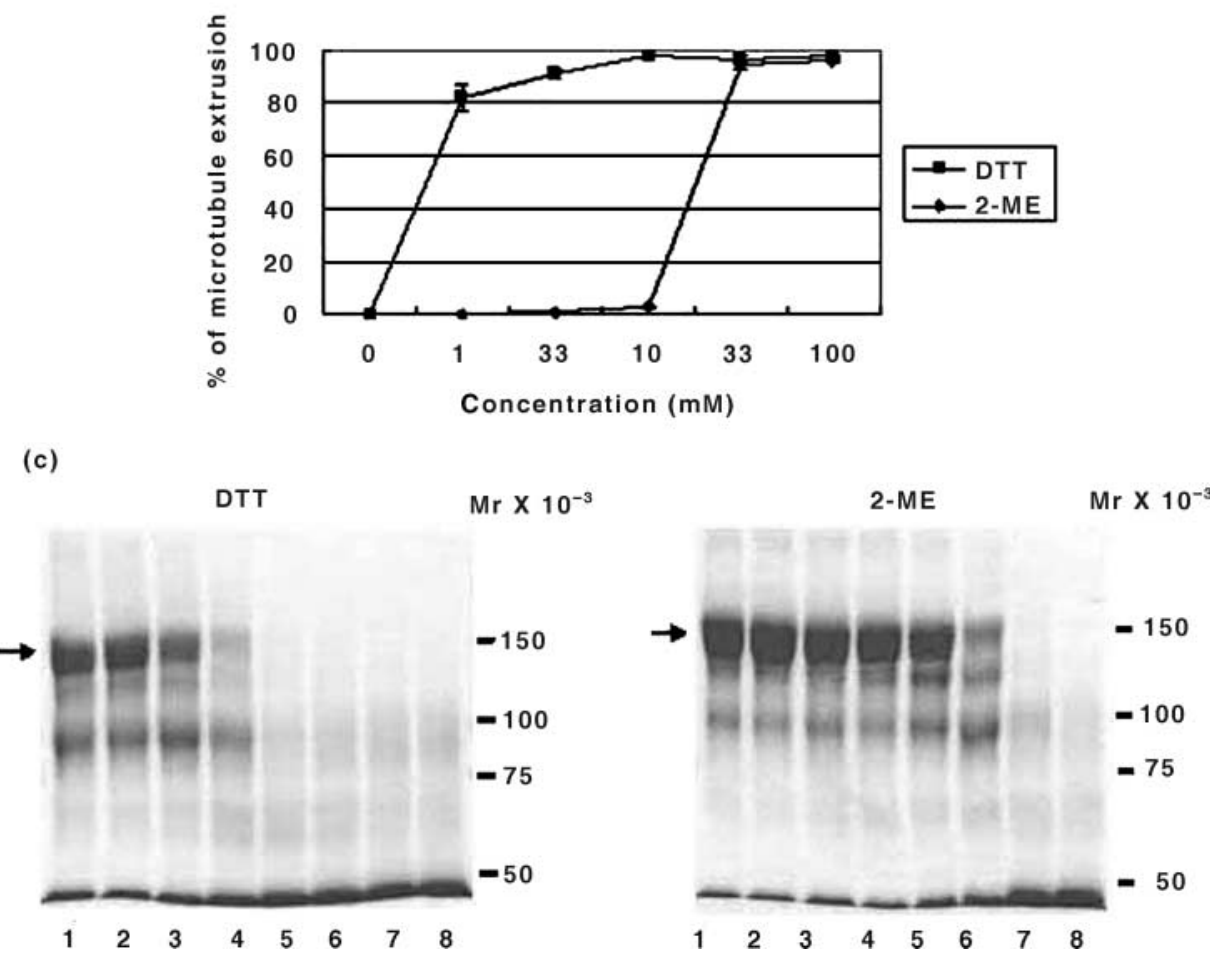

Figure 1 Effects of reducing agents on microtubule extrusion and reduction of the disulfide bonds in IgG. Hamster (a) and mouse (b) spermatozoa were treated with different concentrations of DTT or 2-ME in the reactivation medium, and the percentages of spermatozoa that had extruded microtubules were determined. Each experiment was conducted three times, and 100 spermatozoa were examined for each replicate at each concentration. The values are expressed as the mean \pm S.E.M. (c) SDS-PAGE analysis of IgG for determination of the reducing power of DTT or 2-ME. IgG was incubated for $10 \mathrm{~min}$ at $37^{\circ} \mathrm{C}$ in reactivation medium that contained no additives (control; lane 1), or that contained $0.1 \mathrm{mM}$ (lane 2), $0.3 \mathrm{mM}$ (lane 3), $1 \mathrm{mM}$ (lane 4), $3.3 \mathrm{mM}$ (lane 5), $10 \mathrm{mM}$ (lane 6), $33 \mathrm{mM}$ (lane 7), and $100 \mathrm{mM}$ (lane 8) DTT or 2-ME. After incubation, the samples were denatured in Laemmli's SDS sample buffer without 2-ME and separated on SDS-PAGE. Arrows indicate the dense band of intact IgG. The relative molecular masses are shown on the right. 
Table 1 Percentages of sperm that extrude microtubules upon treatment with trypsin plus DTT or DTT alone

\begin{tabular}{|c|c|c|c|c|}
\hline & $\begin{array}{c}\text { No. of } \\
\text { experiments }\end{array}$ & $\begin{array}{c}2 \mu \mathrm{g} / \mathrm{ml} \\
\text { trypsin plus } \\
1 \mathrm{mM} \text { DTT }\end{array}$ & $1 \mathrm{mM}$ DTT & $33 \mathrm{mM}$ DTT \\
\hline Hamster & $3^{\mathrm{a}}$ & $1.3 \pm 1.5^{b}$ & $4.3 \pm 2.3$ & $92.0 \pm 2.1$ \\
\hline Mouse & 3 & $42.0 \pm 1.9$ & $82.3 \pm 4.8$ & $96.3 \pm 0.3$ \\
\hline
\end{tabular}

${ }^{\mathrm{a}}$ One hundred spermatozoa were examined for each replicate. ${ }^{\mathrm{b}}$ The values are expressed as the mean \pm SEM.

the reducing powers of DTT and 2-ME using IgG as the substrate. As shown in Figure 1c, the dense $150 \mathrm{kDa}$ electrophoretic band, which corresponds to the intact form of IgG, was eliminated by treatment with concentrations greater than $1 \mathrm{mM}$ DTT or $10 \mathrm{mM} 2-\mathrm{ME}$. This result confirms that the disulfide bonds in IgG are reduced by treatment with DTT or 2-ME, and that the effective concentration of $2-\mathrm{ME}$ is 10 -fold higher than that of DTT.

\section{Characteristics of microtubule extrusion induced by reducing agents}

The process of microtubule extrusion was closely observed by phase-contrast microscopy of the spermatozoa of hamsters. The extrusion process was similar for both species in the presence of $33 \mathrm{mM}$ DTT. Microtubules were always extruded from the midpiece region, especially around the head-midpiece and midpiece-principal piece junctions (Fig. 2a and 2b). Extrusion appeared to be on the same side as, and/or the opposite side of the curve of, the spermatozoal heads. Thus, extrusion occurred in the same plane as that in which flagellar bending occurs in intact spermatozoa (Aoki et al. 1994). Extrusion always began on only one side and was followed by extrusion on the other side; extrusion never occurred simultaneously on both sides (Fig. 3a). In some spermatozoa, intrusion, i.e. return to the normal position within the flagellar axoneme, of some extruded microtubules was observed when microtubules were extruded on the opposite side (Fig. 3b).

\section{Discussion}

This is the first report demonstrating that microtubule extrusion is induced efficiently in demembranated spermatozoa following treatment with a reducing reagent alone. Based on previous studies, proteases such as trypsin (Summers \& Gibbons 1973, Brokaw \& Simonick 1977, Olson \& Linck 1977, Si \& Okuno 1993, 1995) and elastase (Brokaw 1980, Ishijima et al. 2002) were considered to be useful inducers of microtubule extrusion. However, in the current study, we have shown that treatment with a reducing agent is effective in inducing microtubule extrusion, and that trypsin has a rather detrimental effect on this process. Since the concentrations of the reducing agents required for the induction of microtubule extrusion corresponded to those sufficient to reduce the disulfide bonds of IgG (Fig. 1c), we speculate that microtubule extrusion is induced by the reduction of disulfide bonds in the proteins that connect the microtubules. The percentage of spermatozoa that (a) Hamster

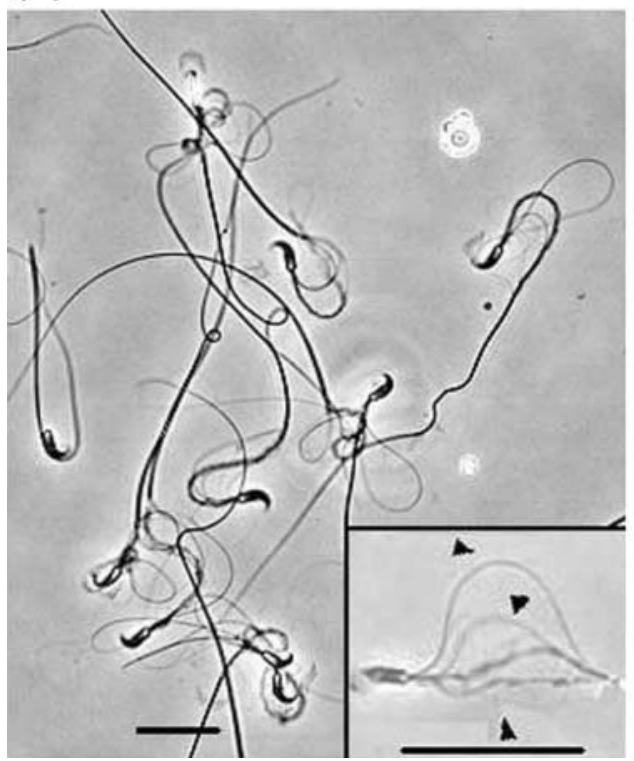

(a) Mouse

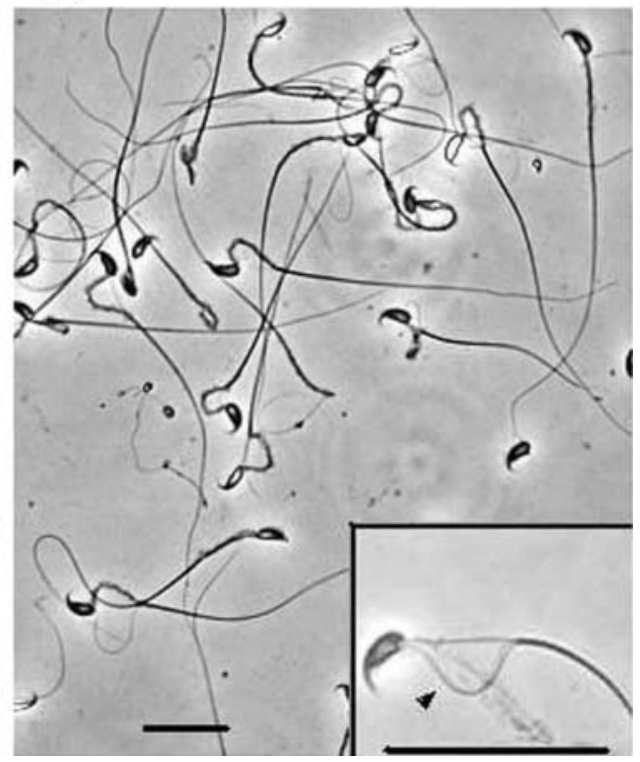

Figure 2 Phase-contrast micrographs of microtubule extrusion. In most of the hamster (a) and mouse (b) spermatozoa, microtubules are extruded from the flagellar axonemes. The spermatozoa were photographed after incubation for $10 \mathrm{~min}$ in reactivation medium that contained $33 \mathrm{mM}$ DTT. Microtubule extrusion appears on the same side as and/or the opposite side of the curve of the spermatozoal heads in the midpiece region; extruded microtubules are indicated by arrowheads in the lower right corner of each image. Details of the methods used for the induction of microtubule extrusion are described in the Materials and Methods section. Scale bars: $20 \mu \mathrm{m}$. 
(a)
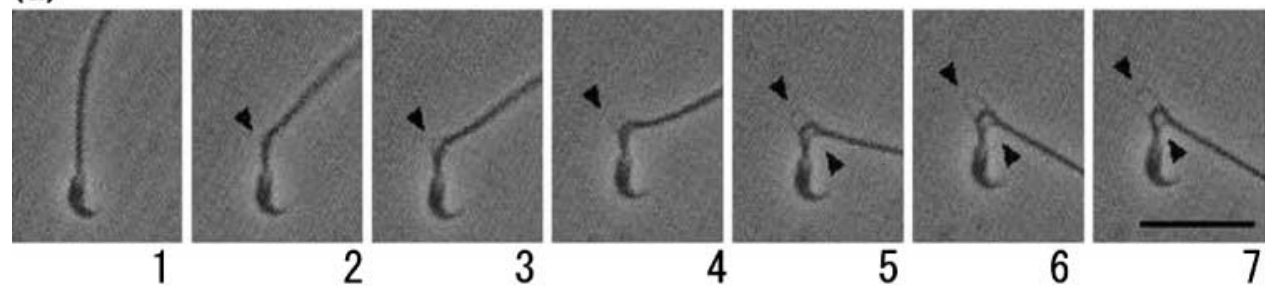

(b)
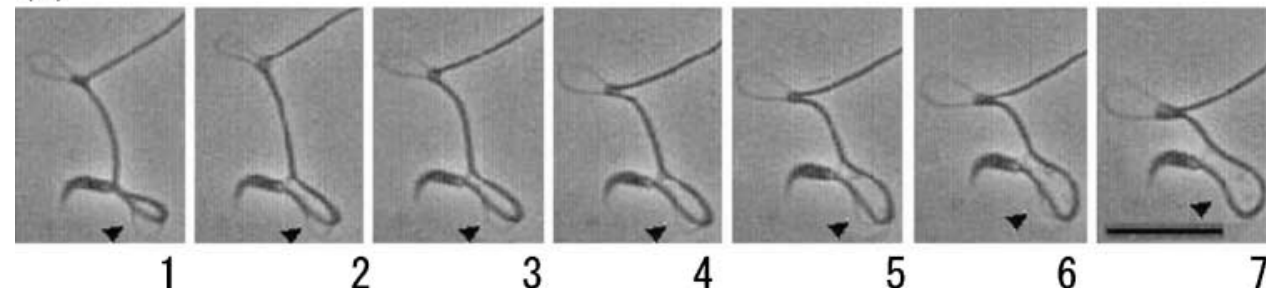

Figure 3 Phase-contrast microscopy images of microtubules extruded from the flagellar axonemes in hamster spermatozoa. (a) Seven consecutive images were collected at six frames per second. The microtubules that are undergoing extrusion are indicated by arrowheads. (b) Seven consecutive images were collected at the rate of six frames every five seconds. A microtubule that is undergoing intrusion is indicated by the arrowhead. Scale bar: $20 \mu \mathrm{m}$.

extruded microtubules was much higher after treatment with DTT alone than after treatment with trypsin plus DTT (Table 1). In DTT-treated spermatozoa, microtubule extrusion and intrusion, which were observed over a long period of time (Fig. 3), occurred alternately. However, in the trypsin-treated spermatozoa, the intrusion of microtubules never occurred after the microtubules were extruded. These results suggest that protease treatment digests one or more regulatory proteins involved in microtubule sliding, as well as proteins that connect the microtubules. Since the present method is likely to produce minimal digestion of axonemal proteins, it should find application in investigations of proteins that regulate motility, such as the microtubule-binding proteins.

The occurrence of intrusion as well as extrusion of microtubules (Fig. 3b) suggests the bidirectional force generation of dynein arms in the flagellum. Electron microscopic observation of trypsin-treated Tetrahymena cilia revealed that dynein arms generate force in only one direction, i.e. from the base to tip (Sale \& Satir 1977). However, our observations suggest that flagellar microtubules slide in two directions, from base to tip and from tip to base, and that dynein arms generate forces in both directions. These differences in generated force direction may account for the different patterns of bending observed between cilia and flagella. The ciliary beat consists of two components, the power stroke and the return stroke, while the flagellar beat consists of an undulating wave that propagates from base to tip (Jahn \& Votta 1972).

The mechanism that regulates alternate bending in the same and opposite directions as the curve of the spermatozoal head remains unclear. Nevertheless, the 'switchpoint' hypothesis, originally advocated by Satir (Satir 1985, Holwill \& Satir 1994) offers an explanation for this phenomenon. This hypothesis proposes that the active bundles of the dynein arms switch, in an alternating fashion, from one side of the flagellum to the opposite side of the flagellum. The mechanistic framework of this hypothesis was explained in the Geometric Clutch hypothesis proposed by Lindemann (1994). When dynein-tubulin bridges form, they produce an adhesive force between adjacent doublets. The bridges pull adjacent doublets slightly closer together, and attachment of the bridges increases the chance for the neighboring dynein heads to also attach to each other. Conversely, detachment of the bridges increases the probability of further detachment of neighboring dynein heads by decreasing the total adhesive force that holds the doublets together. Thus, active dynein engagement on one side of the axoneme inhibits engagement on the opposing side, which results in an alternating switching of the active dyneins. In support of this theory, the bundles of doublets 9,1 , and 2 or those of doublets 5 , 6 , and 7 are usually extruded, whereas both groups of the ATP-disintegrated macrocilia of the ctenophore Beroe are not extruded (Tamm \& Tamm 1984). Nakano et al. (2003) used electron microscopy to show that only one of the two opposed microtubule bundles (the 5-7 or 4-7 bundle, or the 9-2 or 9-3 bundle) was displaced in sea urchin spermatozoa that extruded microtubules after treatment with trypsin. These results suggest that only those microtubules that are located on one side of the flagellum are activated for sliding at any given moment, while those on the other side are inactive, thereby producing an alternating pattern. Furthermore, Lindemann et al. (1992) showed that microtubule bundle 9-2, which produces a bending force in the direction opposite to that of the curve of the head, was extruded in demembranated rat sperm when the flagella were asymmetrically bent in the opposite direction of the curve. Our results also support the 'switch-point' hypothesis: that microtubules could be extruded from the same 
side as that of the curve of the spermatozoal heads, as well as from the opposite side (Fig. 2). Furthermore, the extrusion always began on only one side, and was followed by extrusion on the other side, but never occurred simultaneously on both sides (Fig. 3), which lends support to the hypothesis by Lindemann (1994) that active dynein engagement on one side of the axoneme inhibits engagement on the opposing side.

In this paper, we have shown that microtubule extrusion in mammalian spermatozoa can be induced by treatment with reducing reagents. Compared with the previously established method using proteases, this new method yields a much higher percentage of sperm that extrude microtubules. In addition, our method minimizes the digestion of the structural proteins of the flagellar axoneme and of proteins that are involved in the regulation of flagellar bending. Therefore, this method should prove useful in further investigations of the regulatory mechanisms of microtubule sliding.

\section{Acknowledgements}

We thank Dr Makoto Okuno of the University of Tokyo for helpful discussions and encouragement.

\section{References}

Aoki F, Ishida K, Okuno M \& Kohmoto K 1994 Analysis of flagellar bending in hyperactivated hamster and mouse spermatozoa. Journal of Reproduction and Fertility 101 397-403.

Brokaw CJ 1980 Elastase digestion of demembranated sperm flagella. Science 207 1365-1367.

Brokaw CJ \& Simonick TF 1977 Motility of triton-demembranated sea urchin sperm flagella during digestion by trypsin. The Journal of Cell Biology 75 650-665.

Holwill ME \& Satir P 1994 Physical model of axonemal splitting. Cell Motility and the Cytoskeleton 27 287-298.

Ishijima S \& Witman GB 1991 Demembranation and reactivation of mammalian spermatozoa from golden hamster and ram. Methods in Enzymology 196 417-428.

Ishijima S, Iwamoto T, Nozawa S \& Matsushita K 2002 Motor apparatus in human spermatozoa that lack central pair microtubules. Molecular Reproduction and Development 63 459-463.

Jahn TL \& Votta JJ 1972 Locomotion of protozoa. Annual Review of Fluid Mechanics 4 93-116.

Katz DF, Cherr GN \& Lambert H 1986 The evalution of hamster sperm motility during capacitation and interaction with ovum vestments in vitro. Gamete Research 14 333-346.
Kinukawa M, Nagata M \& Aoki F 2003 Changes in flagellar bending during the course of hyperactivation in hamster spermatozoa. Reproduction 125 43-51.

Laemmli UK 1970 Cleavage of structural proteins during the assembly of the head of bacteriophage T4. Nature 227 680-685.

Lindemann CB 1994 A model of flagellar and ciliary functioning which uses the forces transverse to the axoneme as the regulator of dynein activation. Cell Motility and the Cytoskeleton 29 141-154.

Lindemann CB \& Gibbons IR 1975 Adenosine triphosphate-induced motility and sliding of filaments in mammalian sperm extracted with Triton X-100. Journal of Cell Biology 65 147-162.

Lindemann CB, Orlando A \& Kanous KS 1992 The flagellar beat of rat sperm is organized by the interaction of two functionally distinct populations of dynein bridges with a stable central axonemal partition. Journal of Cell Science 102 249-260.

Nakano I, Kobayashi T, Yoshimura M \& Shingyoji C 2003 Centralpair-linked regulation of microtubule sliding by calcium in flagellar axonemes. Journal of Cell Science 116 1627-1636.

Olson GE \& Linck RW 1977 Observation of the structural components of flagellar axonemes and central pair microtubules from rat sperm. Journal of Ultrastructure Research 61 21-43.

Sale WS \& Satir P 1977 Direction of active sliding of microtubules in Tetrahymena cilia. PNAS 74 2045-2049.

Satir P 1985 Switching mechanisms in the control of ciliary motility. In Modern Cell Biology, pp 1-46. Ed. B Satir. New York, USA: AR Liss.

Shingyoji C, Murakami A \& Takahashi K 1977 Local reactivation of Triton-extracted flagella by iontophoretic application of ATP. Nature 265 269-270.

Si Y \& Okuno M 1993 The sliding of the fibrous sheath through the axoneme proximally together with microtubule extrusion. Experimental Cell Research 208 170-174.

Si Y \& Okuno M 1995 Extrusion of microtubule doublet outer dense fibers 5-6 associating with fibrous sheath sliding in mouse sperm flagella. Journal of Experimental Zoology 273 355-362.

Suarez SS 1988 Hamster sperm motility transformation during development of hyperactivation in vitro and epididymal maturation. Gamete Research 19 51-65.

Suarez SS, Katz DF, Owen DH, Andrew JB \& Powell RL 1991 Evidence for the function of hyperactivated motility in sperm. Biology of Reproduction 44 375-381.

Summers KE \& Gibbons IR 1973 Effect of trypsin digestion on flagellar structures and their relationship to motility. The Journal of Cell Biology 58 618-629.

Tamm SL \& Tamm S 1984 Alternate patterns of doublet microtubule sliding in ATP-disintegrated macrocilia of the ctenophore Beroe. The Journal of Cell Biology 99 1364-1371.

Received 20 May 2004

First decision 13 July 2004

Revised manuscript received 19 August 2004

Accepted 26 August 2004 